\title{
Specific elimination of coxsackievirus B3 infected cells with a protein engineered toxin-antitoxin system
}

\author{
Jung-Ho Park ${ }^{1, *}$, Jin-Ho Park ${ }^{2, *}$, Wonho Choi ${ }^{1}$ \& Byung-Kwan Lim ${ }^{2}$ \\ Received: 11 December 2018 / Accepted: 24 January 2019 \\ (C) The Korean Society of Toxicogenomics and Toxicoproteomics and Springer Nature B.V. 2019
}

\begin{abstract}
Backgrounds: Coxsackievirus B3 (CVB3) is a member of the family Picornaviridae, and along with polioviruses, belongs to the Enterovirus genus. The CVB3 genome is composed single-stranded RNA encoding polyproteins, which are cleaved to individual functional proteins by $2 \mathrm{~A}$ and $3 \mathrm{C}$ proteases proteins which have been targeted for drug development. Here, we showed that protease activity required to activate a toxic protein may be used to prevent viral infection.

Methods: We modified the MazE-MazF antitoxin-toxin system of Escherichia coli to fuse a C-terminal fragment of MazE to the N-terminal end of toxin MazF with a linker having a specific protease cleavage site for CVB3. This fusion protein formed a stable dimer and was capable of inactivating the mRNA interferase activity of MazF which cleaves the ACA sequence in mRNA substrates.
\end{abstract}

Results: The incubation of $2 \mathrm{~A}$ proteases with the fusion proteins induced cleavage between the MazE and MazF fragments from the fusion proteins; the subsequent release of MazF significantly inhibited virus replication. Additionally, we note that, CVB3 infected HeLa cells quickly died through a MazF toxin mediated effect before virus protein expression.

Conclusion: These findings suggest that the MazEF fusion protein has a strong potential to be developed as an anti-virus therapy following CVB3 infection.

\footnotetext{
${ }^{1}$ Bio-Evaluation Center, Korea Research Institute of Bioscience and Biotechnology, Cheongju 28120, Republic of Korea

${ }^{2}$ Department of Biomedical Science, Jungwon University, Goesan-gun, Chungbuk 28024, Republic of Korea

*These authors contributed equally to this work.

Correspondence and requests for materials should be addressed to B. K. Lim( $\varangle$ bklim@jwu.ac.kr)
}

Keywords: Coxsackievirus, MazEF, Antitoxin, Cardiotropic, Polyprotein, Proteases

\section{Introduction}

CoxsackievirusB3 (CVB3) is a cardiotropic virus that binds to the coxsackievirus adenovirus receptor to infect host cells ${ }^{1-3}$. CVB3 is a member of the family Picornaviridae, and along with polioviruses, it belongs to the Enterovirus genus. Although most enterovirus infections are subclinical, acute myocardial inflammation triggered by these infections can induce severe arrhythmias and sudden cardiac death or may lead to the development of chronic myocarditis and dilated cardiomyopathy ${ }^{4-10}$. We previously reported several antiviral drugs developed from small chemical compounds. An inhibitor of enterovirus protease $3 \mathrm{C}(3 \mathrm{CPI})$ showed a particularly strong antiviral effect in a murine viral myocarditis model. It significantly reduced viral replication in the heart and minimized myocardial damage ${ }^{11}$.

The proteases encoded by RNA viruses, such as Corona virus, hepatitis A virus (HAV), and CVB3, play an essential role in viral infection, as they are required for the processing of virus-encoded polyproteins ${ }^{12}$. Most bacteria have the toxin-antitoxin (TA) system which is a mechanism to control the cell growth. Toxin induces cell growth arrest and even cause cell death without the co-expression their cognate antitoxins ${ }^{13,14}$. Thus, virus proteases have been considered ideal drug targets. In the present report, we attempted to positively use the activity of the viral proteases to activate a latent toxin (MazF) of Escherichia coli from a toxin-antitoxin (MazF-MazE) fusion protein by cleaving off antitoxin MazE fragment. MazF thus released functions as an ACA-specific mRNA interferase $\mathrm{e}^{15}$ to eliminate almost all cellular mRNA as well as single-stranded viral RNA in the vi- 


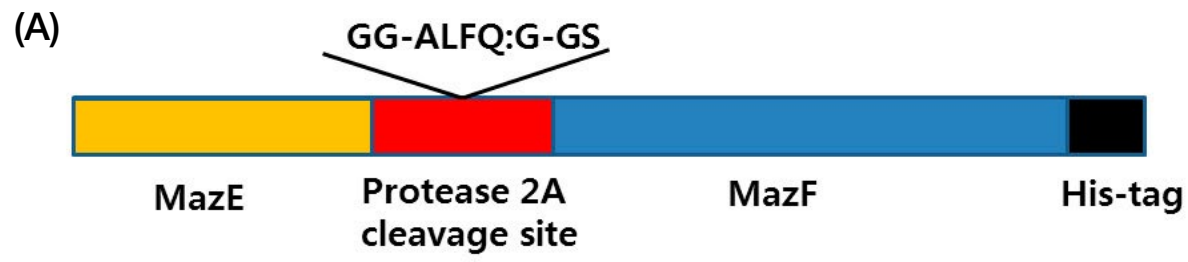

(B)

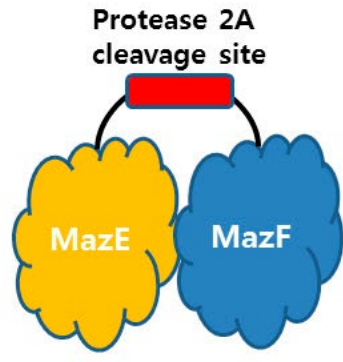

MazF inactive

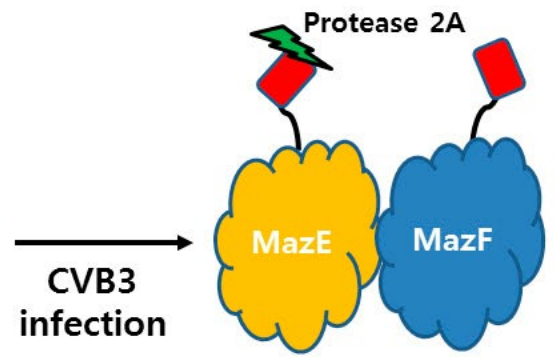

MazF active

Figure 1. Schematic diagram of the MazEF fusion system. The MazEF fusion protein was generated with a linker containing a specific CVB3 protease 2A cleavage site. (A) Schematic of the MazEF fusion protein including the linker. (B) Schematic of the activation of the MazEF fusion protein with a protease cleavage site within the linker.

rus-infected cells.

The CVB3 genome, consisting of a positive single strand RNA 7400 bases, encodes three major polyproteins $(\mathrm{P} 1, \mathrm{P} 2, \mathrm{P} 3)$ which require processing by the CVB3 protease, $2 \mathrm{~A}$. As these viral proteases cleave polyproteins at highly specific amino acid sequences (AXXQ:G), these specific protease cleavage sites for individual RNA viruses may be incorporated into the linker between MazE antitoxin and MazF toxin of the MazE-MazF fusion proteins (MazEF) so that viral proteases induced upon infection cleave the linker to activate MazF as an ACA-specific mRNA interferase. MazF has been shown to be a potent toxin, effectively causing apoptotic cell death in mammalian cells ${ }^{16}$. In $E$. coli, two dimers of MazF form a stable heterohexamer complex with one MazE dimer in the center ${ }^{17,18}$ (Figure $1)$.

In this study, we developed a new enterovirus antiviral system using bacteria to drive the anti-toxin and toxin system. The MazEF toxin significantly inhibited virus replication with viral protease $2 \mathrm{~A}$ activating the bacterial MazEF toxin. Here, we also note that CVB3 infected HeLa cells were quickly destroyed through a MazEF toxin effect before virus protein expression. These findings suggest that MazEF has the potential to be developed as an anti-viral drug for CVB3 infections.

\section{Materials \& Methods}

\section{Virus and cell lines}

CVB3 was derived from an infectious cDNA copy of the cardio tropic $\mathrm{H} 3$ variant of CVB3. Virus titers were determined using the plaque-forming assay in HeLa cells as described previously. HeLa cells were maintained in Dulbecco's Modified Eagle Medium(DMEM, WELGEN Inc, Gyeongsan-si, KOR) supplemented with $10 \%$ fetal bovine serum. HeLa-UVM cells were obtained from Dr. ES Jeon (Samsung Medical Center, Seoul, Korea) ${ }^{19}$.

\section{Construction of protease $2 \mathrm{~A}$ cleaved MazEF fusion proteins}

We previously generated a MazEF fusion protein with a linker contain a specific cleavage site for $\mathrm{HCV}$ proteases (Figure 1A). We have modified the specific cleavage site between $\mathrm{C}$-terminal region of MazE (MazEA1-41) and MazF genes from E. coli for CVB3 protease 2A and added four extra residues (Gly-Gly and Gly-Ser at the N-terminal and the C-terminal ends, respectively) (Figure $1 \mathrm{~B})^{18}$.

\section{In vitro HeLa cell survival assay}

We generated a MazEF working system into green fluorescence protein plasmid (pEGFP) for the in vitro cell 
(A)
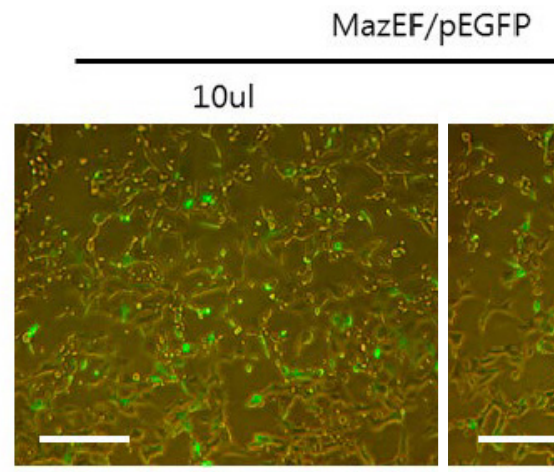

$20 u l$

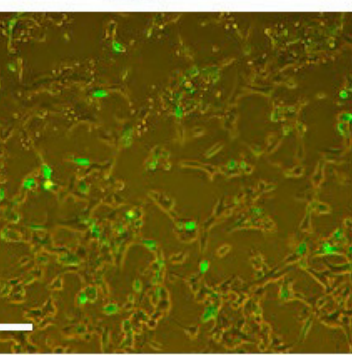

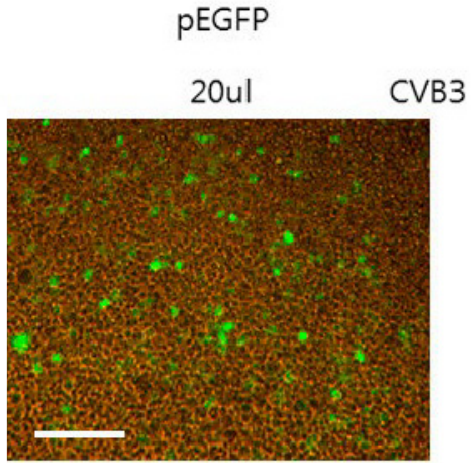

(B)

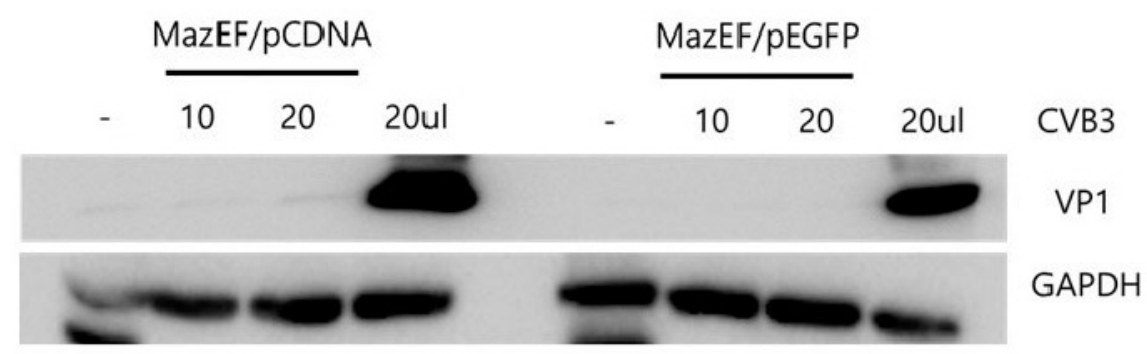

Figure 2. MazEF expression inhibited CVB3 replication in HeLa cells. (A) MazEF expression significantly suppressed CVB3 replication. MazEF-GFP expressing HeLa cells quickly died following CVB3 infection induced cytopathic effect. (B) Viral capsid protein VP1 expression was completely blocked by MazEF expression with CVB3 infection. CVB3 replication was significantly inhibited by MazEF expression (scale bar, $100 \mu \mathrm{m}$ ).

survival assay. In brief, HeLa cells were transfected with the MazEF/pEGFP plasmid for 24 hours followed by addition of CVB3 at multiplicity of infection (m.o.i.) of $1(10 \mu \mathrm{L})$ and $2(20 \mu \mathrm{L})$. After 12 hours of infection, we observed the impact on cell morphology and cytopathic effects using fluorescence microscope, and extracted protein for western blot analysis.

\section{Western blot analysis}

Cells were lysed in RIPA buffer $(50 \mathrm{mM}$ Tris- $\mathrm{HCl}$, pH 8.0, 0.1\% SDS, $1 \%$ NP40, $150 \mathrm{mM} \mathrm{NaCl}, 0.5 \%$ sodium deoxycholate). Aliquots $(10 \mu \mathrm{g})$ of total cell extracts were loaded onto $10 \%$ SDS-PAGE gels. Following gel electrophoresis, proteins were transferred to Hybond-ECL nitrocellulose membranes (Amersham Biosciences, Piscataway, NJ, USA). The membranes were blocked in 5\% non-fat dry milk solution in phosphate-buffered saline (PBS) containing $0.1 \%$ Tween-20 and incubated with anti-enterovirus VP1 (1 : 1000, mouse monoclonal; Novocastra, Lincolnshire, IL, USA), eIF4G1 (1 : 1000, rabbit polyclonal; Cell Signaling Technology, Danvers, MA, USA), and GAPDH antibodies $(1: 1000$, rabbit polyclonal antibodies; Cell Signaling Technology, Danvers, MA, USA) $)^{19}$.

\section{Statistics}

The data are presented as the mean \pm SEM. Differences in measured parameters between control and target experimental groups were examined using the MannWhitney U t-test (GraphPad Prism 3.0 for Windows; GraphPad Software, La Jolla, CA, USA). Survival rates were analyzed using the Kaplan-Meier method. $P<0.05$ was considered significant.

\section{Results}

\section{MazEF induce virus infected cell death}

To confirm the antiviral effect of the MazEF toxin, we have overexpressed GFP conjugated MazEF (MazEF/ pEGFP) or MazEF only (MazEF/pcDNA3). After 12 hours, CVB3 was added into the cell culture plate and to confirm the cytopathic effect on HeLa cells. As shown in Figure 2A, MazEF-GFP expressing HeLa cells indicate MazEF toxin expression and these cells experienced a greater cytopathic effect in a virus concentration dependent manner compare to GFP overexpressed cells. Western blot analysis revealed that MazEF toxin significantly decreased CVB3 replication in CVB3 infected cells, resulting in a reduction in de- 
(A)

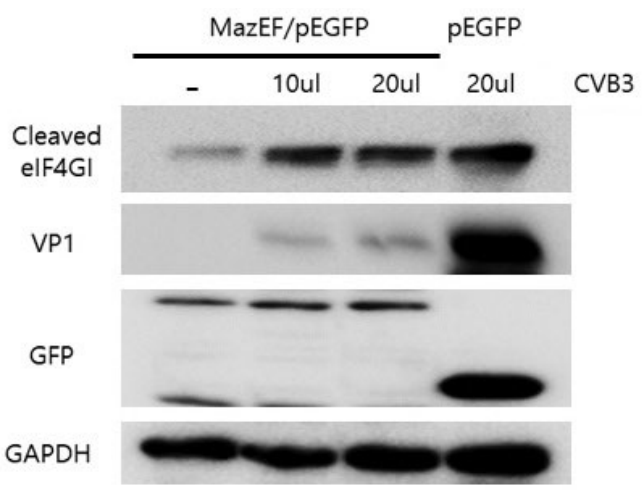

(B)

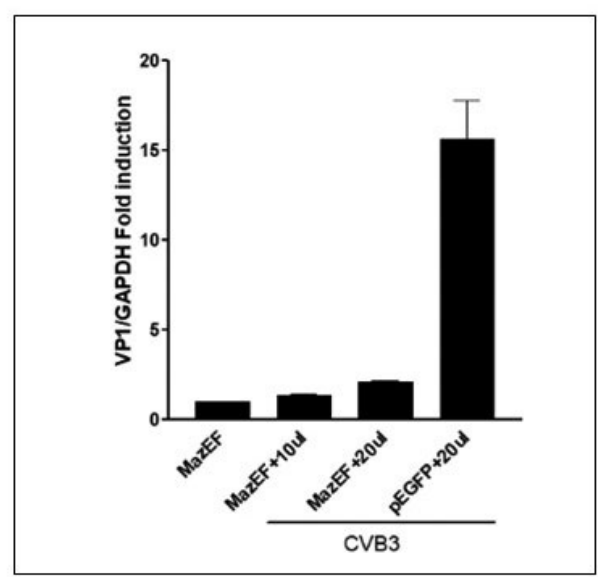

Figure 3. CVB3 protease 2A induce MazEF cell toxicity. (A) CVB3 protease $2 \mathrm{~A}$ expressed in CVB3 infected HeLa cell and then cleaved host transcription initiation factor eIF4G1. MazEF-GFP expression reduced virus capsid protein VP1 expression. Virus replication was significantly inhibited by the MazEF toxin. (B) Data are presented as the mean \pm SEM from 3 independent experiments ( $* * *, P<0.01)$.

tectable virus capsid protein VP1 compare with control cells (Figure 2B).

\section{Target inhibition of virus replication}

The overexpression of MazEF toxin in HeLa cells resulted in rapid cell death and reduced expression of virus capsid protein VP1 in CVB3 infected cells. Translation initiation factor protein eIF4G1 was cleaved by viral protease $2 \mathrm{~A}$ soon after CVB3 infection and inhibited host protein production. As shown in Figure 3, cleaved eIF4G1 was detected in CVB3 infected cells. However, viral capsid protein VP1 was significantly decreased in MazEF toxin treated HeLa cells, because cell death is induced by the MazEF toxin before virus replication. These results demonstrated that CVB3 infected HeLa cells were killed by direct overexpression of MazEF toxin before initiation of virus replication.
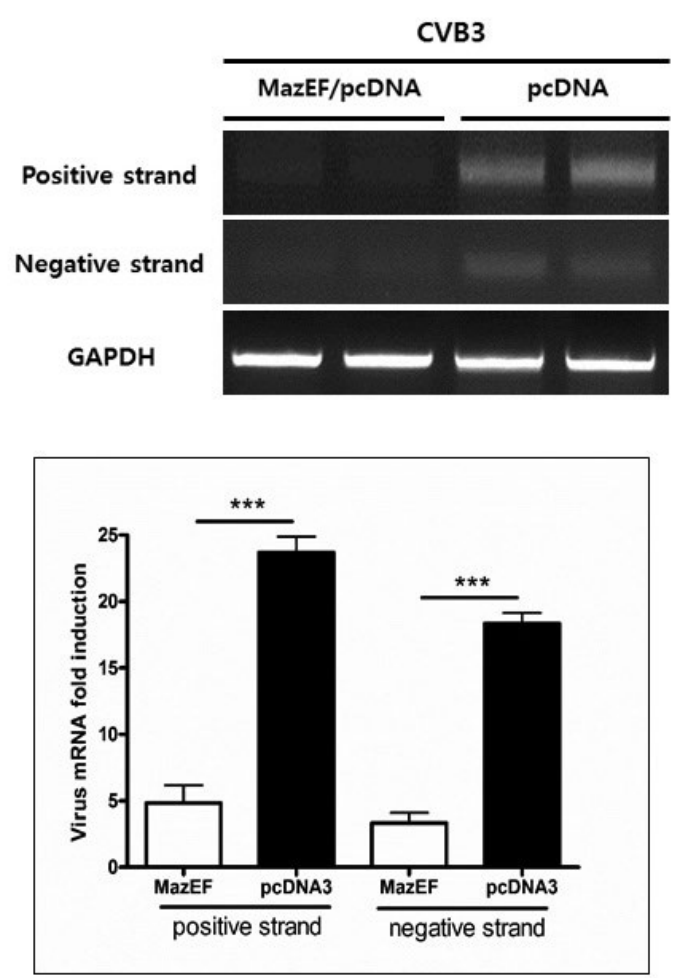

Figure 4. MazEF inhibit CVB3 genome replication. CVB3 protease $2 \mathrm{~A}$ activates the MazEF fusion protein which led to rapid death of HeLa cells infected with CVB3. MazEF expression inhibited the CVB3 capsid protein VP1 positive and negative strand RNA amplification. Virus genome amplification was significantly inhibited by MazEF toxin. Data are presented as the mean \pm SEM from 3 independent experiments $(* * *, P<0.01)$.

\section{MazEF expression inhibit CVB3 genome replication}

Amplification of viral RNA amplification is essential for viral replication. However, MazEF toxin resulted in rapid cell death in virus infected cells. We confirmed the CVB3 capsid protein positive and negative strand RNA replication using RT-PCR. In CVB3 infected HeLa cells, CVB3 genome expression was significantly decreased in cells expressing the MazEF fusion protein compared with control cells (Figure 4). CVB3 protease2A modified the structure of the MazE and MazF proteins. MazEF fusion resulted in the rapid death of CVB3 infected cells which subsequently affects virus genome amplification.

\section{Discussion}

Xiong et al. have previously demonstrated in vitro that the enteroviral protease $2 \mathrm{~A}$ directly cleaves murine dystrophin in the hinge 3 region $^{20}$ in order to determine whether this cleavage plays an important role in 
the development of enterovirus-mediated myocarditis, sarcolemma membrane disruption, viral propagation and direct virus-mediated cytopathic effect in vivo ${ }^{21}$. In this study, we applied this concept to inhibit virus propagation and systemic side effects accompanying in CVB3 infection. The first stage of viral replication involves processing of the protease $2 \mathrm{~A}$ into a polyprotein; this is a critical time point to inhibit virus amplification and propagation to the other cells.

Since these viral proteases cleave polyproteins at highly specific amino acid sequences, these specific protease cleavage sites for individual RNA viruses may be incorporated into a linker between the MazE antitoxin and MazF toxin of the MazE-MazF fusion protein so that viral proteases induced upon infection cleave the linker to activate MazF as an ACA-specific mRNA interferase. Activation of MazF in mammalian cells has been result in BAK-dependent apoptotic cell death $^{16}$. We could regulate CVB3 infected cell death through MazEF fusion protein induction by CVB3 expressed protease $2 \mathrm{~A}$. In addition, the fusion protein may potentially be used as a tool for the prevention and/or treatment of RNA virus infections, as cells carrying the fusion protein with a linker cleavable with a specific RNA viral protease may attain resistance to a RNA viral infection ${ }^{18}$. However, the MazEF fusion protein in vivo systemic effect is not clear and additional research is required to better elucidate this.

These results suggest that the new application for CVB3 protease 2A induction of bacterial toxin protein, MazEF, is applicable to the treatment of many single-strand RNA virus infections, (eg, CVB3 myocarditis, enterovirus71, and influenza virus). MazEF induction was confirmed in CVB3 infected HeLa cells and it was strongly suppressed virus replication and genome amplification. Therefore, the MazEF toxin system could be beneficial in the treatment of cardiomyopathy associated with enterovirus infection.

\section{Conclusion}

CVB3 is the main cause of viral myocarditis and meningitis in childhood. However, effective antiviral drugs have not yet been developed. Our TA system leveraging the MazEF fusion protein, is a potential targeted antiviral system. MazEF activation is regulated by target virus protease expression and may be a potential therapeutic target in human CVB3 infections.

Acknowledgements Jung-Ho Park and Jin-Ho Park contributed equally to this study. This study was supported by grants from the National Research Foundation (NRF) of Korea provided by the Korean Government (No. NRF-
2016R1D1A1A02937046, Lim BK), the Next-Generation BioGreen 21 Program (Project No. PJ01368601), Rural Development Administration, Republic of Korea, and the KRIBB Research Initiative Program.

Conflict of Interest Jung-Ho Park, Jin-Ho Park, Wonho Choi, \& Byung-Kwan Lim declares that they have no conflict of interest.

Human and animal rights The article does not contain any studies with human and animal and this study was performed following institutional and national guidelines.

\section{References}

1. Lim, B. K. et al. Coxsackievirus and adenovirus receptor (CAR) mediates atrioventricular-node function and connexin 45 localization in the murine heart. J Clin Invest 118, 2758-2770 (2008).

2. Baboonian, C., Davies, M. J., Booth, J. C. \& McKenna, W. J. Coxsackie B viruses and human heart disease. Curr Top Microbiol Immunol 223, 31-52 (1997).

3. Huber, S. A. \& Lodge, P. A. Coxsackievirus B-3 myocarditis. Identification of different pathogenic mechanisms in DBA/2 and Balb/c mice. Am J Pathol 122, 284-291 (1986).

4. Feldman, A. M. \& McNamara, D. Myocarditis. N Engl J Med 343, 1388-1398 (2000).

5. Herskowitz, A., Beisel, K. W., Wolfgram, L. J. \& Rose, N. R. Coxsackievirus B3 murine myocarditis: wide pathologic spectrum in genetically defined inbred strains. Hum Pathol 16, 671-673 (1985).

6. Knowlton, K. U., Jeon, E. S., Berkley, N., Wessely, R. \& Huber, S. A mutation in the puff region of VP2 attenuates the myocarditic phenotype of an infectious cDNA of the Woodruff variant of coxsackievirus B3. $J$ Virol 70, 7811-7818 (1996).

7. Knowlton, K. U. \& Badorff, C. The immune system in viral myocarditis: maintaining the balance. Circ Res 85, 559-561 (1999).

8. Martino, T. A., Liu, P. \& Sole, M. J. Viral infection and the pathogenesis of dilated cardiomyopathy. Circ Res 74, 182-188 (1994).

9. Xiong, D. et al. Inducible cardiac-restricted expression of enteroviral protease $2 \mathrm{~A}$ is sufficient to induce dilated cardiomyopathy. Circulation 115, 94-102 (2007).

10. Woodruff, J. F. Viral myocarditis. A review. Am J Pathol 101, 425-484 (1980).

11. Yun, S. H. et al. Antiviral activity of coxsackievirus B3 $3 \mathrm{C}$ protease inhibitor in experimental murine myocarditis. J Infect Dis 205, 491-497 (2012).

12. Lim, B. K. et al. Foreign gene transfer to cardiomyocyte using a replication-defective recombinant coxsackievirus B3 without cytotoxicity. Intervirology 55, 201-209 (2012).

13. Choi, W. et al. Translation-dependent mRNA cleavage 
by YhaV in Escherichia coli. FEBS Letters 591, 18531861 (2017).

14. Choi, W., Yoon, M. H. \& Park, J. H. Functional Characterization of the C-Terminus of YhaV in the Escherichia coli PrlF-YhaV Toxin-Antitoxin System. $J$ Microbiol Biotechnol 28, 987-996 (2018).

15. Zhang, Y. et al. MazF cleaves cellular mRNAs specifically at ACA to block protein synthesis in Escherichia coli. Mol Cell 12, 913-923 (2003).

16. Shimazu, T. et al. NBK/BIK antagonizes MCL-1 and BCL-XL and activates BAK-mediated apoptosis in response to protein synthesis inhibition. Genes \& Development 21, 929-941 (2007).

17. Kamada, K., Hanaoka, F. \& Burley, S. K. Crystal structure of the MazE/MazF complex: molecular bases of antidote-toxin recognition. Mol Cell 11, 875-884 (2003).
18. Park, J. H., Yamaguchi, Y. \& Inouye, M. Intramolecular regulation of the sequence-specific mRNA interferase activity of MazF fused to a MazE fragment with a linker cleavable by specific proteases. Appl Environ Microbiol 78, 3794-3799 (2012).

19. Lim, B. K. et al. Soluble coxsackievirus B3 3C protease inhibitor prevents cardiomyopathy in an experimental chronic myocarditis murine model. Virus Res 199, 1-8 (2015).

20. Xiong, D. et al. Dystrophin deficiency markedly increases enterovirus-induced cardiomyopathy: a genetic predisposition to viral heart disease. Nat Med 8, 872-877 (2002).

21. Lim, B. K. et al. Inhibition of Coxsackievirus-associated dystrophin cleavage prevents cardiomyopathy. $J$ Clin Invest 123, 5146-5151 (2013). 